\title{
The Manchester Respiratory Activities of Daily Living Questionnaire: Reliability and Validity of the Chinese Version with Pictorial Enhancement
}

This article was published in the following Dove Press journal: International Journal of Chronic Obstructive Pulmonary Disease

Damian Chi Hong Siu, (iD ${ }^{1,2}$

Chi Tao So, ${ }^{1,3}$ Cherry Wai Lee Lau, (iD) 1,4 Eric Hei Man Hui, (D 1,5 Alexandra Fung, 1,6 Tak Ming Chan, ${ }^{1,7}$ Fion Siu Fun Chan, ${ }^{1,3}$ Catherine Kam Fung Chan, ${ }^{1,8}$ William Pang Wai Chen, ${ }^{1,9}$ Sammy Hoo Ming Cheung, 1,10 Katherine Pui Yee Chan, I, II Peter Poon, (iD) ${ }^{12}$ Michael Cheung, ${ }^{12}$ Cherry Chan, ${ }^{12}$ Justin Tse, ${ }^{12}$ Vanessa Fung, ${ }^{12}$ Kenneth NK Fong (D) 12

'Respiratory Sub-Specialty Group, Occupational Therapy Central Organization Committee, Hospital Authority, Hong Kong SAR, ${ }^{2}$ Occupational Therapy Department, Prince of Wales Hospital, Hospital Authority, Hong Kong SAR, ${ }^{3}$ Occupational Therapy Department, Princess Margaret Hospital, Hospital Authority, Hong Kong SAR,

${ }^{4}$ Occupational Therapy Department, Kowloon Hospital, Hospital Authority, Hong Kong SAR, ${ }^{5}$ Occupational Therapy Department, Grantham Hospital, Hospital Authority, Hong Kong SAR,

${ }^{6}$ Occupational Therapy Department, Ruttonjee Hospital, Hospital Authority, Hong Kong SAR, ${ }^{7}$ Occupational Therapy Department, Haven of Hope Hospital, Hospital Authority, Hong Kong SAR, ${ }^{8}$ Occupational Therapy Department, Wong Tai Sin Hospital, Hospital Authority, Hong Kong SAR, 'Occupational Therapy Department, Tseung Kwan O Hospital, Hospital Authority, Hong Kong SAR, ${ }^{10}$ Occupational Therapy Department, Tuen Mun Hospital, Hospital Authority, Hong Kong SAR, "Occupational Therapy Department, Alice Ho Miu Ling Nethersole Hospital, Hospital Authority, Hong Kong SAR,

${ }^{12}$ Department of Rehabilitation Sciences, The Hong Kong Polytechnic University, Hong Kong SAR
Correspondence: Damian Chi Hong Siu Occupational Therapy Department, Hospital Authority, Prince of Wales Hospital, 30-32 Ngan Shing Street, Shatin, New Territories, Hong Kong SAR

Email damiansiu@gmail.com
Background: The Manchester Respiratory Activities of Daily Living Questionnaire (MRADLQ) is a valid and reliable tool measuring the functional level of patients with COPD in multidimensional aspects. However, a local validation of the questionnaire is lacking in Hong Kong.

Objective: To develop a Chinese version of MRADLQ with pictorial enhancement (C-MRADLQ) and study its reliability and validity.

Patients and Methods: A total of 238 patients suffering from COPD were recruited from nine public hospitals and five Nurse and Allied Health Respiratory Clinics by convenient sampling. A total of 64 patients with normal spirometry results and no previous clinical diagnosis of COPD were invited to complete the C-MRADLQ for comparison and examination of its validity. Ten out of 302 patients were re-assessed with the C-MRADLQ after one week by the same rater for test-retest reliability. The C-MRADLQ was correlated with spirometry result, COPD classifications and groups by Global Initiative for Chronic Obstructive Lung Disease (GOLD), the modified Medical Research Council Dyspnea Scale (mMRC Dyspnea Scale), COPD Assessment Test (CAT), Chinese Version of the Shortness of Breath Questionnaire (C-SOBQ), number of admission and the ADO index.

Results: The C-MRADLQ shows good test-retest reliability as indicated by an intra-class correlation coefficient value of 0.975 . It is significantly correlated with COPD stage, COPD group, SOBQ score, CAT score, mMRC, ADO index, spirometry results, and number of admissions. The SOBQ score, number of admissions, FEV1/FVC, and COPD group could significantly predict the total C-MRADLQ score. A total of $67.9 \%$ of participants' mMRC levels were correctly classified by using the C-MRADLQ total score. The agreement of the original and new versions of questions 20 and 21 of C-MRADLQ was $97.3 \%$ and $90.1 \%$, respectively.

Conclusion: The pictorial version of the C-MRADLQ is a validated and reliable functional assessment tool to measure functional status among patients with COPD in the Chinese population.

Keywords: pulmonary disease, chronic obstructive, patient-reported outcome measures, activities of daily living, validation study, occupational therapy

\section{Introduction}

Chronic Obstructive Pulmonary Disease (COPD) is one of the most prevalent causes of morbidity and mortality in the world. ${ }^{1}$ With disease progression, there is a pronounced reduction in functional status and exercise capacity, and an increase in dyspnea and fatigue, which have a negative impact on Health-Related Quality of Life (HRQoL) and activities of daily living (ADL). ${ }^{2-4}$ Eventually, some COPD patients become partially or totally limited in activity of daily living (ADL). ${ }^{5}$ 
Traditional subjective evaluations of COPD patients were mainly focused on perception of symptoms/dyspnea, Health-Related Quality of Life (HRQoL), COPD knowledge, awareness, and attitude. However, these may not entirely reflect how the functional status of patients is influenced by COPD, which is crucial for health-care professionals in planning rehabilitation and care intervention as well as evaluation of treatment outcome. ${ }^{6}$ Therefore, measurement of functional status of patients with COPD is essential in a multidimensional evaluation for health-care professionals to have a comprehensive picture of the impacts of the disease on patients. The Chinese version of the Shortness of Breath Questionnaire (C-SOBQ) with pictorial enhancement is a valid and reliable instrument which gives precise information about the impact of dyspnea on functional activities for patients. ${ }^{7}$ However, it does not evaluate the functional status of these patients.

The Manchester Respiratory Activities of Daily Living Questionnaire (MRADLQ) is a multidimensional patientreported questionnaire which is simple to use, responsive to therapy, and sensitive enough to detect changes. ${ }^{8,9}$ MRADLQ is a self-administered scale originally launched in English and takes about 10 minutes to complete. It has been translated into Portuguese. ${ }^{4}$ It is reliable and valid, showing high internal consistency (Cronbach's Alpha = 0.91). ${ }^{2}$ In predicting patients' functioning, a lower score in the MRADLQ indicates patients are encountering more difficulties in ADL, and a score $\leq 7.5$ is considered a predictor of mortality. ${ }^{10}$ The scoring system ranges from 0 to 21, with higher scores indicating better physical functioning in daily activities. It measures functional ability in four domains: (1) mobility (seven items); (2) kitchen (four items); (3) domestic tasks (six items); and (4) leisure activities (four items). It provides specific information in formulating a care plan to maintain an individual's independent living. It discriminates well between COPD and normal healthy elderly and is responsive to pulmonary rehabilitation. ${ }^{10}$ However, local validation of the questionnaire is lacking in the Hong Kong and Chinese populations.

Therefore, the objectives of the study were: (a) to develop a Chinese version of MRADLQ with pictorial enhancement (C-MRADLQ) which is reliable and valid to measure ADL functions of COPD patients, (b) to examine its criterion-related validity with other related instruments of clinical parameters, (c) to examine its discriminant validity among COPD patients with different levels of severity, and (d) to examine its test-retest reliability.

\section{Methods}

This was an observational cross-sectional study in which data were collected through interviews and medical notes review. The study employed a convenience sample of 238 patients suffering from COPD of different severities according to GOLD classification (Global Initiative for Chronic Obstructive Lung Disease, 2019). Participants were recruited from nine public hospitals and five Nurse and Allied Health-led respiratory clinics between July and September 2020. They were required to meet the inclusion criteria: (1) diagnosed with COPD with a ratio of forced expiratory volume in one second (FEV1) to forced vital capacity (FVC) less than 70\%; (2) medically stable (no admission related to respiratory problems at least four weeks prior to assessment). Participants were excluded based on the following criteria: (1) with active exacerbation of COPD; (2) with current or recurrent symptomatic ischemic heart disease; (3) with lung cancer; (4) with known psychiatric illness; (5) with active tuberculosis; and (6) with marked orthopedic problem limiting ADL. Sixty-four patients with normal spirometry results, no previous clinical diagnosis of COPD, medically stable attending consultation at Nurse and Allied Health Respiratory clinics were invited to complete MRADL for comparison and to examine its validity. They were excluded if (1) with current or recurrent symptomatic ischemic heart disease; (2) with lung cancer; (3) with known psychiatric illness; (4) with active tuberculosis; and (5) with marked orthopedic problem limiting ADL.

\section{Design}

To study the test-retest reliability of the C-MRADLQ, ten out of the 302 patients were selected by convenience sampling from one of the convalescent hospitals for testretest reliability of the C-MRADLQ. The reassessment was completed within one week by the same rater. The two sets of results were then analyzed. The content validity of the instrument, instructions, and testing procedures of the C-MRADLQ were verified by a panel comprised of one expert from Hong Kong Polytechnic University and 15 occupational therapists with extensive experiences in working with COPD patients in public hospitals. Agreement was obtained from the original authors and the study's expert panel. Items score of C-MRADLQ from the 302 patients were used to examine the internal 
consistency. To establish criterion validity, the selfadministered C-MRADLQ was correlated with seven related instruments and clinical parameters in four domains: (1) lung function; (2) GOLD classification and score of the modified Medical Research Council Dyspnea Scale (mMRC Dyspnea Scale); (3) self-administered symptoms in COPD Assessment Test (CAT) and Chinese Version of the Shortness of Breath Questionnaire (C-SOBQ); and (4) combined risk and symptoms assessment in terms of COPD group and ADO index. The parameters were collected from the medical records of each selected patient. If those results were not available, assessments were conducted with a standard assessment protocol by trained occupational therapy students under the direct supervision of registered occupational therapists.

\section{Ethical Approval}

The study complied with ethical principles for medical research as described in the Helsinki Declaration. Ethical approval was obtained from Cluster research committees for seven clusters from the Hospital Authority and the local research ethics committee in the Hong Kong Polytechnic University as listed in the supplementary. All participants provided written informed consent after an explanation of procedures and that they had the right to withdraw from the study at any time.

\section{Instruments}

\section{Chinese MRADLQ}

The Chinese MRADLQ has 21 items under four categories of activities including mobility, kitchen tasks, domestic tasks, and leisure activities. The rating are in four scales: Not at all to alone easily; Much more slowly to Not at all more slowly; Most of the night to Not at all. The overall scoring in each question is regrouped to scoring 0 (Not at all or with help) and scoring 1 (on my own or on my own with mild difficulty). In the Portuguese version, some of the activity items were adapted according to the local culture with the consent of the authors. ${ }^{4}$

For this self-administered Chinese version of MRADLQ, the items were first validated by a focus group of COPD patients. The scoring scale was modified into a six-point scale in order to facilitate measurement of patient progress. In questions 1 to 19 , the original category "Alone with difficulty" was further elaborated $(0=$ Not at all; $1=$ Need help; 2 = Alone with severe difficulty; $3=$ Alone with moderate difficulty; $4=$ Alone with mild difficulty; 5 = Alone easily). In question 20, the mid- range of slowness was introduced $(0=$ much more slowly; $1=$ quite a lot more slowly; $2=$ quite slow; 3 = moderately slow; 4 = a little more slowly; $5=$ Not at all). The whole scale of question 21 was replaced by percentage of sleeping time involved $(0=$ Most of the night; $1=$ About $80 \%$ of time; $2=$ About $60 \%$ of time; $3=$ About $40 \%$ of time; 4 $=$ About $20 \%$ of time; $5=$ Not at all).

In order to improve C-MRADLQ sensitivity, the scoring scale was expanded from four to six choices and the maximum score was 105 . To enhance cultural relevancy, the expert panel recommended modifications to the questions on "wash and dry yourself" and "manage your own garden" to "carry out personal hygiene" and "general exercise", with similar metabolic equivalent (METs). The scale of two questions "Question 20: Do you have to eat more slowly than you would like?" and "Question 21: Does your breathing keep you awake at night?" were updated to facilitate patients' understanding and measurement of progress.

A pictorial enhancement e-form version was developed by a group of occupational therapy students to facilitate administration and understanding of test items. Patients were invited to complete the original and pictorial enhancement e-form simultaneously to examine the agreement between the two versions (Figure 1).

\section{Chinese SOBQ}

The function of the Chinese version of the Shortness of Breath Questionnaire (C-SOBQ) is to assess the level of dyspnea related to ADL among patients. The C-SOBQ was scored by summing responses across all 24 items to form a total score ranging from 0 to 120 . The Chinese version of the SOBQ with pictorial enhancement demonstrated significant correlations with the mMRC Dyspnea Scale, GOLD COPD classifications, BODE index, BMI, and $6 \mathrm{MWD}{ }^{7}$

\section{The Modified Medical Research Council (mMRC) Dyspnea Scale}

The Modified Medical Research Council (mMRC) Dyspnea Scale is a five point scale (0 to 4 ) that considers a single dimension (ie, physical task such as walking) that provokes breathlessness. ${ }^{11}$

\section{The COPD Assessment Test (CAT)}

This is a patient-completed questionnaire assessing all aspects of the impact of COPD (cough, sputum, breathlessness, chest tightness, confidence, activity, sleep, and 
The Chinese version of Manchester Respiratory ADL Questionnaire

\begin{tabular}{|l|l|l|l|l|}
\hline Name: & \multicolumn{2}{|l|}{ Date } & \\
\hline Gender / Age: & & & OPD / HOSP no. & \\
\hline
\end{tabular}

The Manchester Respiratory Activities of Daily Living Questionnaire

This questionnaire is designed to give us a better idea of how your breathing problems are affecting you in your daily life.

\begin{tabular}{|c|c|c|c|c|c|}
\hline Not at all & With help & $\begin{array}{c}\text { Alone with } \\
\text { severe } \\
\text { difficulty }\end{array}$ & $\begin{array}{c}\text { Alone with } \\
\text { moderate } \\
\text { difficulty }\end{array}$ & $\begin{array}{c}\text { Alone with } \\
\text { mild difficulty }\end{array}$ & Alone easily \\
\hline $\mathbf{0}$ & $\mathbf{1}$ & $\mathbf{2}$ & $\mathbf{3}$ & $\mathbf{4}$ & $\mathbf{5}$ \\
\hline
\end{tabular}
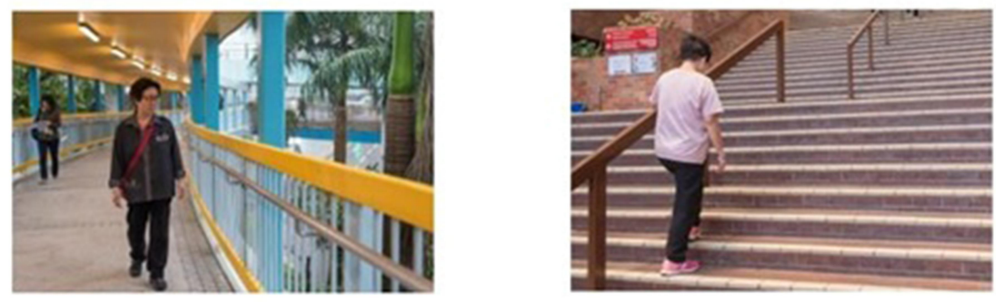

\section{Walk outside the house?}

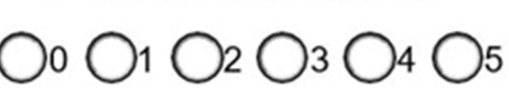

\section{Climb stairs?}

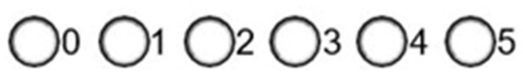

MRADL TOTAL

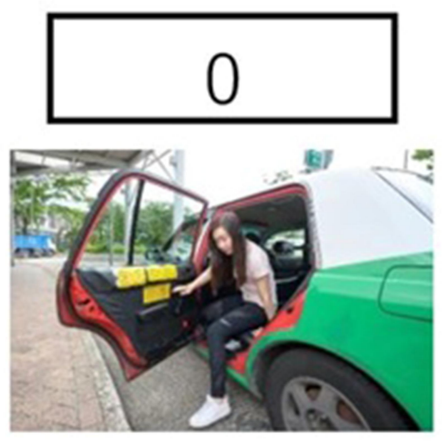

3. Get in and out of the car?

Figure I The Chinese version of the Manchester Respiratory Activities of Daily Living Questionnaire (C-MRADLQ) with pictorial enhancement (modified in English for submission).

energy levels). ${ }^{12}$ There are eight questions on a 1 to 5 point scale.

\section{ADO Index}

The ADO index refers to Age (A), Dyspnea (D), and Obstruction (O); it is a simple multidimensional 15 point scale $(0-14)$ in which a higher score indicates a higher risk of death. ${ }^{13}$

\section{Spirometry}

Patients attending the assessment who had spirometry results within one year were assessed according to the ATS/ERS Standardization Guideline. Forced expiratory volume in the first second (FEV1), Forced Vital Capacity (FVC), and their predicted values were collected, adjusting for their age, gender, body height, and weight and race. ${ }^{14}$

\section{Statistical Analysis}

Data analysis was conducted using IBM SPSS Statistics 25.0 (SPSS Inc., Chicago, IL). Demographic data and data regarding the COPD severity by spirometry will be presented descriptively and compared using chi-square test. The test-retest reliability of the C-MRADLQ was determined using the intraclass correlation coefficient (ICC; twoway mixed effect model) with a $95 \%$ confidence interval.
Ten participants from the respiratory medical department of a convalescent hospital were chosen to investigate test-retest reliability. ICC was determined based on a predicted value of ICC ranging from 0.8 to 0.9 with a 0.05 confidence level. ${ }^{15}$ Internal consistency was measured using Cronbach's alpha which measured pairwise correlations between tested items. To determine the criterion-related validity, the correlation between self-administered C-MRADLQ and other instruments or clinical measures obtained were evaluated using Spearman's rank correlation coefficient. Percent agreements were calculated to compare the original version and this modified version of C-MRADLQ, as well as questions 20 and 21. Discriminant validities of the self-administered C-MRADLQ were determined using discriminant functional analysis. This was used to determine if the C-MRADLQ could distinguish categorical dependent variables or groupings of COPD patients. The C-MRADLQ score was analyzed with COPD classifications (ie, stages and groups) and mMRC scale as the group memberships. Multiple linear regression analysis using the stepwise method was also used to analyze the relationships between the variables, including the ADO index, COPD classification (stage and group), mMRC scale, CAT, lung function test (FEV1, FVC, FEV1/FVC, FEV1\% predicted, FVC $\%$ predicted), and 
C-SOBQ as predictors of the C-MRADLQ total score. The level of statistical significance was set at $p \leq 0.05$.

\section{Results}

Three hundred and two participants, 282 males and 20 females, were recruited in the analysis of criterion-related and discriminant validity. The mean age of participants was 69.81, ranging from 43 to 91 years. Participants at COPD stage I to IV, mMRC 0 to 4 , and risk of COPD were involved. Two hundred and thirty eight patients with different severities of COPD and 64 patients with normal spirometry results and no previously clinical diagnosis of COPD completed the C-MRADL. In the lung function test, the mean FEV1/FVC was $56.97 \%$ (SD 15.56). The mean score of the ADO index, ranging from 0 to 13, and the mean of the C-MRADLQ score, ranging from 0 to 105 , were 7.10 and 97.69, respectively (Table 1). About half of the COPD patients were Stage I-II, Group A and B, and mMRC Grade 0-1 (Figure 2).

An ICC value of 0.975 was obtained $(p \leq 0.001)$, which indicated good test-retest reliability. ${ }^{16}$ Besides, the Cronbach's alpha score was 0.944 , indicating a high level of internal consistency. ${ }^{16}$ Criterion validity of the C-MRADLQ was examined by correlation with different clinical measures. The total C-MRADLQ score was negatively correlated with COPD stage $(\mathrm{r}=-0.546)$, COPD group $(\mathrm{r}=-0.533)$, SOBQ score $(\mathrm{r}=-0.738)$, CAT score $(\mathrm{r}=-0.534)$, mMRC $(\mathrm{r}=-0.577)$, and ADO index $(\mathrm{r}=$ -0.494 ) (Table 2). In terms of measures of disease severity, the total C-MRADLQ score was significantly correlated with FEV1/FVC, FVC, FEV1, respective predicted values, and number of admissions ( $r=-0.359$ to 0.580$)$. Looking into the domains in C-MRADLQ, significant positive correlations were found between the total score of C-MRADLQ and scores of "Mobility (Q.1-7)" $(\mathrm{r}=0.919)$, "Kitchen (Q.8-11)" ( $\mathrm{r}=0.688)$, "Domestic tasks (Q.12-17)" ( $\mathrm{r}=$ $0.860)$, "Leisure (Q.18-19)" ( $\mathrm{r}=0.654)$ and "Others (Q.20, 21)" ( $\mathrm{r}=0.667)$. However, age and gender had no significant correlation with the C-MRADLQ score.

The results of regression (Table 3 ) indicated that only the SOBQ score, number of admissions, FEV1/FVC, and COPD group could significantly predict the total C-MRADLQ score $(\mathrm{B}=-1.297$ to 1.108 , standard error $(\mathrm{SE})=0.029$ to 0.522 , $\mathrm{t}=-14.593$ to $3.218, \mathrm{p}<0.034)$. All those factors accounted for $63.2 \%$ of the total variance in the total C-MRADLQ score. The final regression equation to predict the total C-MRADLQ score was devised as: C-MRADLQ $=98.916-$ 0.423 (SOBQ score) - 1.297 (number of admission) + 0.101 (FEV1/FVC) + 1.108 (COPD group).

By using the C-MRADLQ total score for predicting the classification of COPD stage, COPD group, and mMRC level (Table 4 ), the results proved that $32.5 \%$ of COPD stage, $42.7 \%$ of COPD group, and $67.9 \%$ of mMRC levels of the participants were correctly classified. Meanwhile, comparing the agreement of original scale and the new version of C-MRADLQ, the overall degree of agreement of question 20 (eating) was $97.3 \%$, while that of question 21 (sleeping) was $90.1 \%$.

Table I Demographic Characteristics of Patients

\begin{tabular}{|c|c|c|c|c|c|c|c|c|}
\hline & \multicolumn{4}{|c|}{ COPD $(n=238)$} & \multicolumn{4}{|c|}{ At Risk (Normal) $(n=64)$} \\
\hline & \multirow[t]{2}{*}{ Mean } & \multirow[t]{2}{*}{ SD } & \multicolumn{2}{|l|}{$95 \% \mathrm{Cl}$} & \multirow[t]{2}{*}{ Mean } & \multirow[t]{2}{*}{ SD } & \multicolumn{2}{|l|}{$95 \% \mathrm{Cl}$} \\
\hline & & & Lower & Upper & & & Lower & Upper \\
\hline Age & 70.37 & 8.80 & 69.25 & 71.49 & 67.73 & 8.91 & 65.51 & 69.96 \\
\hline $\mathrm{FEV}_{\text {। }}(\mathrm{L})$ & 1.80 & 3.94 & 1.32 & 1.48 & 3.28 & 8.36 & 1.19 & 5.36 \\
\hline FVC (L) & 2.68 & 0.81 & 2.58 & 2.79 & 2.94 & 0.73 & 2.76 & 3.12 \\
\hline FEV, /FVC (\%) & 51.69 & 13.03 & 50.02 & 53.35 & 76.60 & 4.66 & 75.43 & 77.76 \\
\hline $\mathrm{FEV}, \%$ predicted (\%) & 58.35 & 23.98 & 55.29 & 61.41 & 91.56 & 16.6 & 87.41 & 95.71 \\
\hline FVC \% predicted (\%) & 83.96 & 22.71 & 81.06 & 86.86 & 90.09 & 14.9 & 86.37 & 93.82 \\
\hline C-MRADLQ total score & 96.30 & 11.34 & 94.85 & 97.75 & 102.84 & 2.61 & 102.19 & 103.48 \\
\hline CAT & 9.01 & 6.38 & 8.20 & 9.83 & 1.39 & 1.96 & 0.90 & 1.88 \\
\hline $\mathrm{mMRC}$ & 1.34 & 0.70 & 1.25 & 1.43 & 0.78 & 0.45 & 0.67 & 0.89 \\
\hline SOBQ & 21.81 & 18.18 & 19.49 & 24.13 & 7.69 & 4.91 & 6.46 & 8.91 \\
\hline ADO index & 7.61 & 2.22 & 7.32 & 7.89 & 5.20 & 1.83 & 4.75 & 5.66 \\
\hline
\end{tabular}

Abbreviations: CAT, COPD Assessment Test; $\mathrm{Cl}$, Confidence Interval; COPD, chronic obstructive pulmonary disease; FEVI, forced expiratory volume in I second; FVC, forced vital capacity; FEVI\% predicted, predicted value of forced expiratory volume in I second; FVC \% predicted, predicted value of forced vital capacity; mMRC, modified Medical Research Council Dyspnea Scale; C-MRADLQ, The Manchester Respiratory Activities of Daily Living; SD, standard deviation; SOBQ, Shortness of Breath Questionnaire. 


\section{Subgroup Analysis of Patients $(n=302)$}

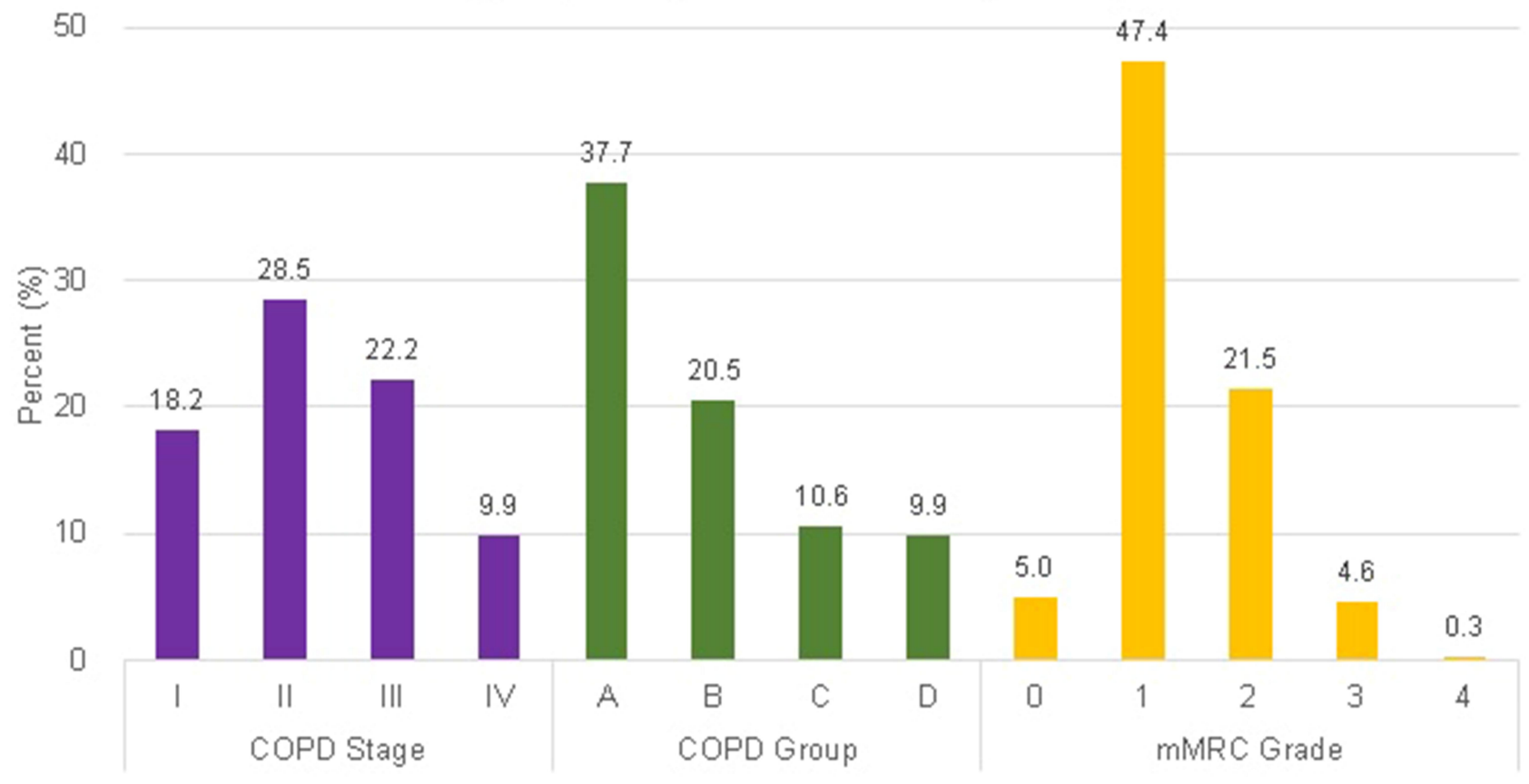

Figure 2 Subgroup analysis of patients $(n=302)$.

Table 5 compares C-MRADLQ total score with COPD stage and group. The results of one-way ANOVA showed that there was a significant main effect of COPD stage on the C-MRADLQ total score $(F(4,297)=48.22, p<0.001$, $\eta p^{2}=0.39$ ). Bonferroni post hoc tests showed that patients with poorer lung function (COPD Stage II-IV) scored significantly lower in C-MRADLQ compared to patients with normal spirometry results. Meanwhile, there was a significant main effect of COPD grouping on the C-MRADLQ total score $(\mathrm{F}(4,297)=29.93, \mathrm{p}<0.001$, $\eta \mathrm{p}^{2}=0.29$ ). Bonferroni post hoc tests showed that patients with more symptoms and history of acute exacerbation leading to hospital admission (COPD Group B to D) scored significantly lower in C-MRADLQ compared to patients with normal spirometry results.

\section{Discussion}

In this study, C-MRADLQ was found to have the strongest correlation with C-SOBQ. C-SOBQ was used to assess self-reported dyspnea associated with specific $\mathrm{ADL},{ }^{17}$ while C-MRADLQ was used to measure COPD patients' functional level in daily activities.

The results suggested that C-MRADLQ was an effective and efficient instrument to assess patients' functional performance affected by dyspnea. In comparison with C-SOBQ, it was found to be sensitive to detect rehabilitation program outcomes. ${ }^{2}$ A moderate correlation was found between C-MRADLQ and GOLD COPD classification, revealing that the level of difficulty in completing daily tasks in C-MRADLQ correlates with the physiological impairment reflected by spirometry lung function tests. The findings agreed with those of a previous study. ${ }^{18}$

A moderate correlation was obtained between the C-MRADLQ and ADO index. Considering the calculation of ADO index, mMRC and lung function were moderately correlated with C-MRADLQ as mentioned above while age was found to have a low correlation with C-MRADLQ. The result is similar to that of some studies, that is age is not significantly correlated to self-reported ADL ability and lung function in COPD. ${ }^{19,20}$ It was interesting to observe in our study that numerous old patients obtained a high score in C-MRADLQ, which meant that they could still perform ADL well. Confounding factors on puff medication and co-morbidity with other chronic diseases cannot be neglected.

A fair correlation was found between C-MRADLQ and number of hospital admissions. Although a higher number of hospital admissions could certainly reflect more severe 
Table 2 Correlation Matrix of C-MRADLQ with Other Criterion Parameters $(n=302)$

\begin{tabular}{|l|l|}
\hline $\begin{array}{l}\text { Criterion-Related } \\
\text { Parameters }\end{array}$ & $\begin{array}{l}\text { Spearman's Rho } \\
\text { Coefficient }\end{array}$ \\
\hline Age & -0.088 \\
Gender & 0.021 \\
COPD stage & $-0.546^{*}$ \\
COPD group & $-0.533^{*}$ \\
Number of admissions & $-0.359^{*}$ \\
SOBQ & $-0.738^{*}$ \\
CAT & $-0.534^{*}$ \\
mMRC & $-0.577^{*}$ \\
Mobility (Q.I-7) & $0.919^{*}$ \\
Kitchen (Q.8-II) & $0.688^{*}$ \\
Domestic tasks (Q.12-17) & $0.860^{*}$ \\
Leisure (Q.I8-19) & $0.654^{*}$ \\
Others (Q.20a,2la) & $0.667^{*}$ \\
FVC & $0.382^{*}$ \\
FVC \% predicted & $0.423^{*}$ \\
FEVI & $0.578^{*}$ \\
FEVI\% predicted & $0.580^{*}$ \\
FEVI/FVC & $0.514^{*}$ \\
ADO index & $-0.494^{*}$ \\
\hline
\end{tabular}

Note: ${ }^{*} \mathrm{p} \leq 0.05$.

Abbreviations: C-MRADLQ, Manchester Respiratory ADL Questionnaire; COPD, chronic obstructive pulmonary disease; SOBQ, Shortness of Breath Questionnaire; FEVI, forced expiratory volume in I second; FVC, forced vital capacity; FEVI\% predicted, predicted value of forced expiratory volume in I second; FVC \% predicted, predicted value of forced vital capacity; mMRC, modified Medical Research Council Dyspnea Scale.

pulmonary symptoms and poorer physical functioning of patients, these patients had received pulmonary rehabilitation before and practiced several adaptive and energy conservation techniques for ADL, such as coping skills and breathing and relaxation techniques. ${ }^{21}$ Thus, they might score higher in C-MRADLQ with less functional limitation in ADL. This phenomenon supports the use of C-MRADLQ as a substantial outcome measure for documenting the progress of patients in the Pulmonary Rehabilitation Program, despite the fact that some patients may not report improvement in their dyspnea levels.

The results of regression demonstrated moderate agreement with the results of correlation analysis (Table 3). The study found that SOBQ, COPD group, FEV1/FVC, and number of admissions were significant predictors of C-MRADLQ total score. All four indices are essential information to be collected before enrolling patients in the pulmonary rehabilitation program. This indicated that ADL functioning, as reflected by C-MRADLQ, could be used as another important marker for patients' classification and stratification in triage for the needs of pulmonary rehabilitation, especially in the presence of more symptoms and recent hospital admission due to acute exacerbation as confirmed by the results of one-way ANOVA (Tables 4 and 5).

When comparing patients with normal spirometry results, C-MRADLQ demonstrated a fair sensitivity and discriminant power for patients with nil or mild functional limitation, especially for those with COPD Stage I and Group A (Table 5). This implied that the 21 scoring items might not be adequately sensitive to detect changes for COPD patients with mild or no symptoms who are independent in daily living tasks. However, large F value and partial eta squared value in Table 5 highlighted a significant main effect of COPD stage and grouping on the C-MRADLQ total score. This led to the conclusion that C-MRADLQ is sensitive to discriminating COPD patients with increased disease severities and symptoms and with recent acute exacerbation history leading to hospitalization. It could be a good self-administered assessment tool to monitor treatment progress and daily living performance after pulmonary rehabilitation or to rectify any puff medication. Meanwhile, the result in predicting mMRC levels suggested that it could be used as a quick screening tool for functionality of COPD patients in different clinical settings.

The scoring in the items of the Activities of Daily Living Inventory (ADLI) reflects the hierarchy of the level of difficulty perceived by patients with COPD. ${ }^{22}$ The agreement between the original and our new version of C-MRADLQ showed that an expanded ordinal scale helps patients to reflect more precisely on their needs, and can be used as a reference for clinicians to select an appropriate treatment for them. For questions 20 and 21 in the new version of C-MRADLQ, the degree of agreement was $97.3 \%$ and $90.1 \%$, respectively. This indicated that the expanded scale of feeding slowness affected by COPD in question 20 could assist patients in rating their actual performance in feeding quality more accurately. In question 21, the wording to describe the extent of sleeping difficulty affected by COPD was changed from actual duration of insomnia into percentage of time involved at night. The percentage of agreement was not as high as that of question 20 but it achieved $90.1 \%$, indicating an acceptable level of agreement compared to the original version of C-MRADLQ. The results supported the fact that our new version of C-MRADLQ is a valid and reliable tool for COPD patients in clinical settings. 
Table 3 Predictions of the C-MRADLQ Total Score $(n=302)$

\begin{tabular}{|l|l|l|l|l|l|l|}
\hline & B & SE & t & P value & $\mathbf{R}^{\mathbf{2}}$ \\
\hline (Constant) & 98.916 & 2.342 & 42.244 & $<0.00 I^{* * *}$ & 0.795 \\
SOBQ & -0.423 & 0.029 & -14.593 & $<0.00 I^{* * *}$ & 0.632 \\
Number of admissions & -1.297 & 0.387 & -3.355 & $0.00 I^{* * *}$ & \\
FEVI/FVC & 0.101 & 0.031 & 3.218 & $0.00 I^{* * *}$ & \\
COPD Group & 1.108 & 0.522 & 2.125 & $0.034^{*}$ & & \\
\hline
\end{tabular}

Notes: ${ }^{*} \mathrm{p} \leq 0.05 ;{ }^{* * *} \mathrm{p}<0.001$.

Abbreviations: COPD, chronic obstructive pulmonary disease; SOBQ, Shortness of Breath Questionnaire.

Table 4 Classification of COPD Stage, COPD Group and mMRC Level Using Total Score of C-MRADLQ $(n=302)$

\begin{tabular}{|c|c|c|c|c|c|c|c|c|c|}
\hline \multirow[t]{2}{*}{ COPD Stage } & \multicolumn{9}{|c|}{ GOLD: Predicted Group Membership, n (\%) } \\
\hline & At Risk of COPD & $\mathbf{I}$ & \multicolumn{2}{|c|}{ II } & \multicolumn{2}{|l|}{ III } & IV & \multicolumn{2}{|c|}{ Total } \\
\hline At risk of COPD & $0(0)$ & $0(0)$ & \multicolumn{2}{|c|}{$64(100)$} & \multicolumn{2}{|c|}{$0(0)$} & $0(0)$ & \multicolumn{2}{|c|}{64} \\
\hline I & $0(0)$ & $0(0)$ & \multicolumn{2}{|c|}{$54(98.2)$} & \multicolumn{2}{|c|}{$\mathrm{I}(\mathrm{I} .8)$} & $0(0)$ & \multicolumn{2}{|c|}{55} \\
\hline II & $0(0)$ & $0(0)$ & \multicolumn{2}{|c|}{$7 I(82.6)$} & \multicolumn{2}{|c|}{$12(14)$} & $3(3.5)$ & \multicolumn{2}{|c|}{86} \\
\hline III & $0(0)$ & $0(0)$ & \multicolumn{2}{|c|}{$40(59.7)$} & \multicolumn{2}{|c|}{$15(22.4)$} & $12(17.9)$ & \multicolumn{2}{|c|}{67} \\
\hline IV & $0(0)$ & $0(0)$ & \multicolumn{2}{|c|}{$7(23.3)$} & \multicolumn{2}{|c|}{$\mathrm{II}(36.7)$} & $12(40)$ & \multicolumn{2}{|c|}{30} \\
\hline \multicolumn{10}{|c|}{$32.5 \%$ of original grouped cases correctly classified } \\
\hline \multirow[t]{2}{*}{ COPD Group } & \multicolumn{9}{|c|}{ COPD Group: Predicted Group Membership, n (\%) } \\
\hline & At Risk of COPD & $\mathbf{A}$ & & B & & C & D & & tal \\
\hline At risk of COPD & $0(0)$ & $64(100)$ & & $0(0)$ & & $0(0)$ & $0(0)$ & 64 & \\
\hline A & $0(0)$ & 108(94.7) & & $4(3$. & & $0(0)$ & $2(1.8)$ & 11 & \\
\hline B & $0(0)$ & $42(67.7)$ & & 12( & 9.4) & $0(0)$ & $8(12.9)$ & 62 & \\
\hline C & $0(0)$ & $21(65.6)$ & & $5(15$ & & $0(0)$ & $6(18.8)$ & 32 & \\
\hline D & $0(0)$ & I4(46.7) & & $7(23$ & & $0(0)$ & $9(30.0)$ & 30 & \\
\hline \multicolumn{10}{|c|}{$42.7 \%$ of original grouped cases correctly classified } \\
\hline \multirow[t]{2}{*}{ mMRC } & \multicolumn{9}{|c|}{ mMRC: Predicted Group Membership, n (\%) } \\
\hline & 0 & $\mathbf{I}$ & & 2 & & 3 & 4 & & Total \\
\hline 0 & $0(0)$ & $30(100)$ & & $0(0)$ & & $0(0)$ & $0(0)$ & & 30 \\
\hline I & $0(0)$ & $183(95.8)$ & & $7(3$. & & $\mathrm{I}(0.5)$ & $0(0)$ & & 191 \\
\hline 2 & $0(0)$ & $44(66.7)$ & & $15(2$ & $2.7)$ & $5(7.6)$ & $2(3$. & & 66 \\
\hline 3 & $0(0)$ & $5(35.7)$ & & $2(1$ & & $6(42.9)$ & $\mathrm{I}(7$. & & 14 \\
\hline 4 & $0(0)$ & $0(0)$ & & $0(0)$ & & $0(0)$ & $\mathrm{I}(10$ & & 1 \\
\hline
\end{tabular}

Abbreviations: COPD, chronic obstructive pulmonary disease; C-MRADLQ, Manchester Respiratory ADL Questionnaire; GOLD, Global Initiative for Chronic Obstructive Lung Disease; mMRC, modified Medical Research Council Dyspnea Scale.

One of our study objectives was to investigate the test-retest reliability of the new version of C-MRADLQ. The ICC value was 0.975 and it showed good test-retest reliability. This implies that the new version of C-MRADLQ is suitable for use as a continuous assessment evaluation tool for occupational therapists to monitor patients' ADL performance and treatment outcomes across time. The expanded ordinal scale in each ADL item provides a more accurate description of ADL performance.

A pictorial CMRADL e-form was developed (Figure 1) and is available. This would facilitate efficient administration when using mobile devices such as mobile phones or tablets, especially for score calculation and documentation. This handy and reliable instrument allows health-care professionals to document ongoing functional status electronically and 
Table 5 Subgroup Analysis of C-MRADLQ Total Score by COPD Stage and Group

\begin{tabular}{|c|c|c|c|c|c|c|c|c|}
\hline & \multirow[t]{2}{*}{$\mathbf{n}$} & \multicolumn{2}{|c|}{ C-MRADLQ Total Score } & \multicolumn{2}{|l|}{$95 \% \mathrm{Cl}$} & \multirow[t]{2}{*}{ F Value } & \multirow[t]{2}{*}{$P$ value } & \multirow[t]{2}{*}{$\eta p^{2}$} \\
\hline & & Mean & SD & Lower & Upper & & & \\
\hline COPD Stage & & & & & & & & \\
\hline Normal & 64 & 102.84 & 2.61 & 102.19 & 103.50 & 48.22 & $<0.001$ & 0.39 \\
\hline Stage I & 55 & 102.55 & 3.35 & 101.64 & 103.45 & & & \\
\hline Stage II & 86 & $99.45 *$ & 6.39 & 98.08 & 100.82 & & & \\
\hline Stage III & 67 & $94.25 * * *$ & 10.05 & 91.80 & 96.71 & & & \\
\hline Stage IV & 30 & $80.40 * * *$ & 17.50 & 73.87 & 86.93 & & & \\
\hline COPD Group & & & & & & & & \\
\hline Group A & 114 & 101.50 & 5.99 & 100.39 & 102.61 & 29.93 & $<0.001$ & 0.29 \\
\hline Group B & 62 & $93.85 * * *$ & 8.79 & 91.62 & 96.09 & & & \\
\hline Group C & 32 & $92.53 * * *$ & 14.64 & 87.25 & 97.81 & & & \\
\hline Group D & 30 & $85.63 * * *$ & 16.35 & 79.53 & 91.74 & & & \\
\hline
\end{tabular}

Notes: Comparing patients with normal spirometry results; ${ }^{*} \mathrm{p} \leq 0.05 ; *^{*} * *_{p}<0.001$.

efficiently among COPD patients in Hong Kong. In addition, the self-administrated e-form would minimize interviewer and recall bias when the patient follows the instructions with photos.

\section{Limitations}

In this cross-sectional study, confounding factors should be taken into account. Information bias on co-morbidity with other chronic diseases and smoking history, such as hypertension, diabetes mellitus or heart diseases were not adjusted and analyzed. Types of short and long acting puff medication and compliance were not collected and analyzed. These factors may affect patients' perception of functional limitation in daily activities. Few patients with severe and high-risk COPD, especially at COPD stage IV and group $\mathrm{C}$ and $\mathrm{D}$, were recruited. Further studies are suggested to recruit more participants with severe and high-risk COPD in the outpatient and hospital settings.

\section{Conclusions}

This study developed a Chinese version of MRADLQ with pictorial enhancement that could be used as a unique local validated functional assessment tool for patients with COPD. It has good test-retest reliability that significantly correlated with COPD stage, COPD group, SOBQ score, CAT score, mMRC, ADO index, spirometry lung function test results, and number of admissions. The SOBQ score, number of admissions, FEV1/FVC, and COPD group could significantly predict the C-MRADLQ total score. Their C-MRADLQ total score correctly classified a total of $67.9 \%$ of mMRC levels of the participants. An e-form was also developed to facilitate efficient administration using mobile devices, especially for score calculation and documentation. This handy and reliable instrument allows healthcare professionals to document daily functional status effectively among patients with COPD in the Hong Kong and Chinese populations.

\section{Acknowledgments}

The authors are grateful for the support of the working group members on Respiratory Care, the Occupational Therapy Coordinating Committee, and the Hospital Authority, for coordination of the study. The author would also like to thank Dr. Maria Leung, Dr. Chik Pui Lee, Dr. Wai Ho Chung, Dr. Shuk Yun Leung, Dr. Lit Ping Chan and Mr. Alex Yue (General Out Patient Clinics under the catchment of New Territories East Cluster), Dr. Yiu Cheong Yeung (Princess Margaret Hospital), Dr. Sai On Ling and Dr. Hoi Yee Kwan (Kowloon Hospital) and Dr. Fan Hon Cheung (Ruttonjee Hospital) for their expertise advice in research design and site coordination.

\section{Disclosure}

The authors report no conflicts of interest in this work.

\section{References}

1. Vogelmeier C, Criner G, Martinez F, et al. Global strategy for the diagnosis, management, and prevention of chronic obstructive lung disease 2017 report. GOLD executive summary. Am J Respir Crit Care Med. 2017;195(5):557-582. doi:10.1164/rccm.201701-0218PP

2. Yohannes AM, Roomi J, Winn S, Connolly MJ. The manchester respiratory activities of daily living questionnaire: development, reliability, validity and responsiveness to pulmonary rehabilitation. $J$ Am Geriatr Soc. 2000;48(11):1496-1500. doi:10.1111/jgs.2000.48.11.1496 
3. Global Initiative for Chronic Obstructive Lung Disease (GOLD). Global strategy for the prevention, diagnosis, management and prevention of COPD. 2019. Available from: http://www.goldcopd.org/. Accessed July 09, 2020.

4. Junkes-Cunha M, Mayer A, Reis C, Yohannes A, Maurici R. The manchester respiratory activities of daily living questionnaire for use in COPD patients: translation into Portuguese and cross-cultural adaptation for use in Brazil. J Bras Pneumol. 2016;42(1):15-21. doi:10.1590/s1806-37562016000000029

5. Miller M, Hankinson J, Brusasco V, et al. Standardisation of spirometry. Eur Respir J. 2005;26(2):319-338. doi:10.1183/ 09031936.05.00034805

6. Palange $\mathrm{P}$, Ward SA, Carlsen $\mathrm{KH}$, et al. Recommendations on the use of exercise testing in clinical practice. Eur Respir J. 2007;29 (1):185-209. doi:10.1183/09031936.00006607

7. Fung A, Chan L, So CT, et al. Reliability and validity of the self-administered Chinese version of the shortness of breath questionnaire (C-SOBQ) in patients with chronic obstructive pulmonary disease. Hong Kong J Occup Ther. 2012;22(2):75-83. doi:10.1016/j. hkjot.2012.12.010

8. Cullen DL, Rodak B. Clinical utility of measures of breathlessness. Respir Care. 2002;47(9):986-993.

9. Mahler DA. Mechanisms and measurement of dyspnea in chronic obstructive pulmonary disease. Proc Am Thorac Soc. 2006;3 (3):234-238. doi:10.1513/pats.200509-103SF

10. Yohannes AM, Baldwin RC, Connoly MJ. Reliability of the Manchester respiratory activities of daily living questionnaire as a postal questionnaire. Age Ageing. 2002;31(5):335-338. doi:10.1093/ageing/31.5.355

11. Fletcher C, Elmes P, Fairbairn A, Wood C. Significance of respiratory symptoms and the diagnosis of chronic bronchitis in a working population. BMJ. 1959;2(5147):257-266. doi:10.1136/ bmj.2.5147.257

12. Jones P, Harding G, Berry P, Wiklund I, Chen W, Kline Leidy N. Development and first validation of the COPD assessment test. Eur Respir J. 2009;34(3):648-654. doi:10.3109/15412555.2013.776920

13. Puhan MA, Hansel NN, Sobradillo P, et al. Large-scale international validation of the ADO index in participants with COPD: an individual participant data analysis of 10 cohorts. BMJ Open. 2012;2(6): e002152. doi:10.1136/bmjopen-2012-002152
14. Ip M, Ko F, Lau A, et al. Updated spirometric reference values for adult Chinese in Hong Kong and implications on clinical utilization. Chest. 2006;129(2):384-392. doi:10.1378/chest.129.2.384

15. Walter SD, Eliasziw M, Donner A. Sample size and optimal designs for reliability studies. Stat Med. 1998;17(1):101-110. doi:10.1002/ (SICI)1097-0258(19980115)17:1<101::AID-SIM727>3.0.CO;2-E

16. Portney LG, Watkins MP. Foundations of Clinical Research: Applications to Practice. 2 ed. Upper Saddle River, NJ: Prentice Hall; 2000.

17. Eakin EG, Kaplan RM, Ries AL, Sassi-Dambron DE. Patients' selfreports of dyspnea: an important and independent outcome in chronic obstructive pulmonary disease. Ann Behav Med. 1996;18(2):87-90. doi:10.1007/bf02909580

18. Peruzza S, Sergi G, Vianello A, Pisent C. Chronic obstructive pulmonary disease (COPD) in elderly participants: impact on functional status and quality of life. Respir Med. 2003;97(6):612-617. doi:10.1053/rmed.2003.1488

19. Benzo RP, Abascal-Bolado B, Dulohery MM. Self-management and quality of life in chronic obstructive pulmonary disease (COPD): the mediating effects of positive affect. Patient Educ Couns. 2016;99 (4):617-623. doi:10.1016/j.pec.2015.10.031

20. Bendixen HJ, Ejlersen Wæhrens E, Wilcke JT, Sørensen LV. Selfreported quality of ADL task performance among patients with COPD exacerbations. Scand J Occup Ther. 2014;21(4):313-320. doi: $10.3109 / 11038128.2014 .899621$

21. Goldstein RS, Guyatt G, Lacasse Y. The components of a respiratory rehabilitation program: a systematic overview. Chest. 1997;111 (4):1077-1088. doi:10.1378/chest.111.4.1077

22. So CT, Man D. Development and validation of an activities of daily living inventory for the rehabilitation of patients with chronic obstructive pulmonary disease. OTJR (Thorofare $N$ J). 2008;28 (4):149-159. doi:10.3928/15394492-20080901-04

International Journal of Chronic Obstructive Pulmonary Disease

\section{Publish your work in this journal}

The International Journal of COPD is an international, peer-reviewed journal of therapeutics and pharmacology focusing on concise rapid reporting of clinical studies and reviews in COPD. Special focus is given to the pathophysiological processes underlying the disease, intervention programs, patient focused education, and self management protocols. This journal is indexed on PubMed Central, MedLine and CAS. The manuscript management system is completely online and includes a very quick and fair peer-review system, which is all easy to use. Visit http://www.dovepress.com/testimonials.php to read real quotes from published authors.

Submit your manuscript here: https://www.dovepress.com/international-journal-of-chronic-obstructive-pulmonary-disease-journal 